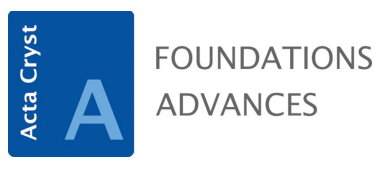

ISSN 2053-2733

Received 24 June 2018

Accepted 27 June 2018

Keywords: perovskites; multiferroics; symmetry.

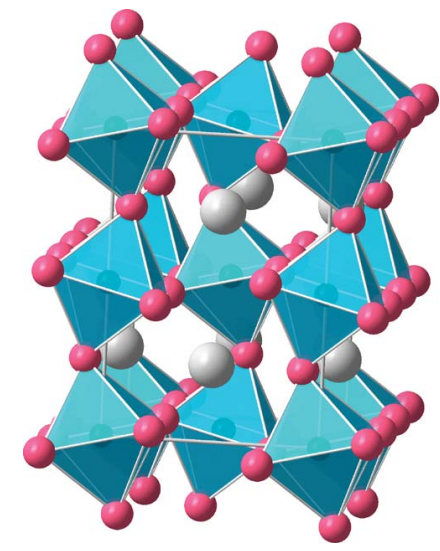

(C) 2018 International Union of Crystallography

\section{A symmetry roadmap to new perovskite multiferroics}

\author{
Patrick M. Woodward*
}

Department of Chemistry and Biochemistry, The Ohio State University, Columbus, OH 43210, USA. *Correspondence e-mail: woodward.55@osu.edu

The cubic perovskite structure is on the surface very simple, with a unit cell that contains only five atoms, all on fixed positions. Yet perovskites exhibit an incredible number of variations on this simple theme. Structural distortions such as octahedral tilting, cooperative Jahn-Teller distortions, polar and antipolar cation displacements are common. Various patterns of chemical ordering can be realized on all three sites of the basic perovskite structure. This compositional diversity and structural flexibility is largely responsible for the large number of important functional materials that come from the perovskite family.

Perovskites are an inviting playground for solid-state materials chemists, but the compositional phase space is so vast that an Edisonian approach to materials discovery is generally not effective. One needs some guidelines - a roadmap, if you will, that shows where to look for the next promising material. Perovskites have long been a source of exceptional dielectric materials, including ferroelectrics $\left(\mathrm{BaTiO}_{3}, \mathrm{PbTiO}_{3}\right)$, relaxor ferroelectrics $\left(\mathrm{PbMn}_{1 / 3} \mathrm{Nb}_{2 / 3} \mathrm{O}_{3}\right)$ and piezoelectrics $\left(\mathrm{PbZr}_{1-x} \mathrm{Ti}_{x} \mathrm{O}_{3}\right)$. More recently, scientists have looked to perovskites for new hybrid improper ferroelectrics - materials where the ferroelectric polarization arises from the combination of two nonpolar lattice distortions, typically polyhedral rotations (Benedek \& Fennie, 2011; Benedek et al., 2015).

Building on pioneering works by Glazer (1972) and Aleksandrov (1976), Howard \& Stokes (1998) were the first to apply group-theory tools to analyze the symmetry consequences of octahedral tilting distortions in perovskites. That work spawned a series of articles that examined the combinations of octahedral tilting with cation ordering (Howard et al., 2003), polar and antipolar cation displacements (Stokes et al., 2002), and Jahn-Teller distortions (Howard \& Carpenter, 2010), among others. In this issue, Mark Senn and Nicholas Bristowe expand this approach to include magnetism, and in the process develop guiding principles for finding new magnetoelectric and multiferroic materials (Senn \& Bristowe, 2018).

Using symmetry arguments, Senn and Bristowe lay out a roadmap for the development of new magnetoelectric perovskites. Their approach starts by looking at how hybrid improper ferroelectricity can arise from coupling of various nonpolar structural distortion mechanisms, patterns of chemical order and/or external lattice strains. Next, they introduce magnetism and show that when the $A$ - and $B$-site cations adopt certain patterns of antiferromagnetic order they can induce ferroelectricity through a trilinear coupling mechanism. Their analysis identifies the compositional space that should be fertile ground for finding new type-II multiferroics, those where both ferroelectricty and ferromagnetism are strongly coupled because magnetic ordering breaks inversion symmetry. They apply similar principles to develop guidelines for finding weak ferromagnetism in perovskites, an important consideration for possible technological applications of multiferroics. Their article culminates by bringing these design principles together to offer guidance on the design of materials that are both ferroelectric and weakly ferromagnetic.

Over the years, chemists have developed good heuristics for predicting when local distortions like Jahn-Teller distortions or local cation off-centering will occur. There is a reasonably good understanding of what combination of ions will produce different types of octahedral tilting or specific patterns of cation ordering. Some distortion combinations that lead to hybrid improper ferroelectricity were previously known, such as layered ordering of $A$-site cations combined with in-phase and out-of-phase $\left(a^{-} a^{-} b^{+}\right)$octahedral 


\section{scientific commentaries}

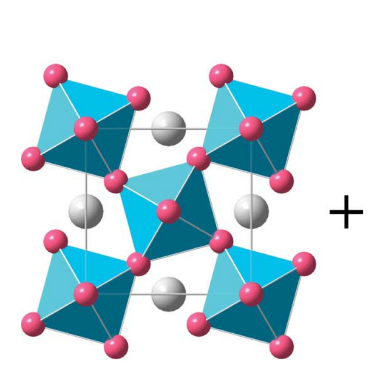

In-phase tilting

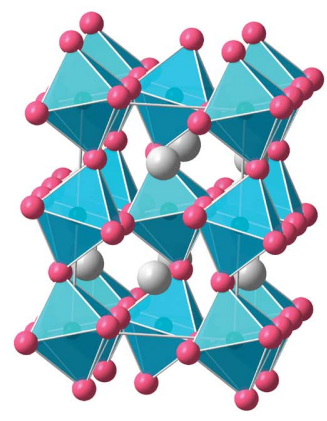

Out-of-phase tilting

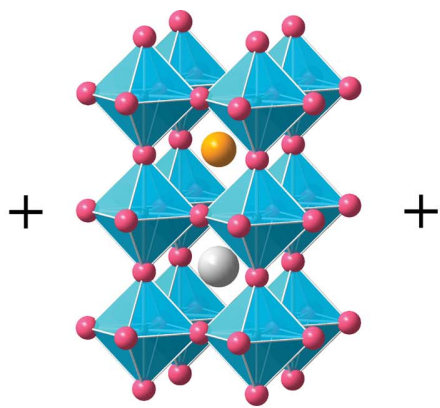

$A$-site cation ordering

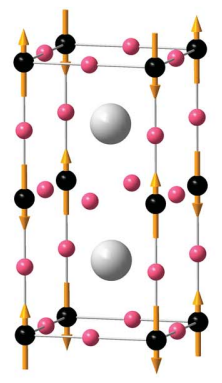

$B$-site magnetic ordering

Figure 1

The combination of in-phase and out-of-phase octahedral tilting, layered ordering of $A$ and $A^{\prime}$ cations, and antiferromagnetic ordering of $B$-site cations is one of many possible recipes for creating perovskites that are simultaneously improper ferroelectrics and weak ferromagnets.

tilting, but this is the first time a comprehensive symmetry analysis of the combined effects of structural distortions, antiferromagnetism and polar distortions has been carried out.

Armed with these new guidelines for designing magnetoelectrics and multiferroics, it is now up to the experimentalists to create materials that have the right combinations of distortions and ordering. For example, to find new materials that exhibit coupled ferroelectricity and weak ferromagnetism Senn and Bristowe advocate searching for perovskites with layered $A$-site cation ordering, $a^{-} a^{-} b^{+}$octahedral tilting and robust antiferromagnetic ordering of $B$-site cations (Fig. 1). Even with these guidelines, finding new multiferroics will not be a walk in the park. In this particular example, it has previously been shown that $A$-site cations tend not to spontaneously adopt a layered ordering pattern unless the $B$ sites are at least partially occupied by non-magnetic $d^{0}$ cations (King \& Woodward, 2010), which makes it difficult to stabilize magnetism at technologically relevant temperatures. Still, knowing where to look is much better than searching in the dark.

\section{References}

Aleksandrov, K. S. (1976). Ferroelectrics, 14, 801-805.

Benedek, N. A. \& Fennie, C. J. (2011). Phys. Rev. Lett. 106, 107204.

Benedek, N. A., Rondinelli, J. M., Djani, H., Ghosez, P. \& Lightfoot, P. (2015). Dalton Trans. 44, 10543-10558.

Glazer, A. M. (1972). Acta Cryst. B28, 3384-3392.

Howard, C. J. \& Carpenter, M. A. (2010). Acta Cryst. B66, 40-50.

Howard, C. J., Kennedy, B. J. \& Woodward, P. M. (2003). Acta Cryst. B59, 463-471.

Howard, C. J. \& Stokes, H. T. (1998). Acta Cryst. B54, 782-789.

King, G. \& Woodward, P. M. (2010). J. Mater. Chem. 20, 5785-5796.

Senn, M. S. \& Bristowe, N. C. (2018). Acta Cryst. A74, 308-321.

Stokes, H. T., Kisi, E. H., Hatch, D. M. \& Howard, C. J. (2002). Acta Cryst. B58, 934-938. 